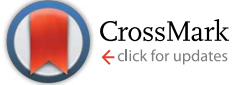

Cite this: RSC Adv., 2015, 5, 1350
Received 4th September 2014 Accepted 26th November 2014

DOI: $10.1039 / c 4 r a 09814 \mathrm{e}$

www.rsc.org/advances

\section{Porphyrin-based electrostatically self-assembled multilayers as fluorescent probes for mercury(II) ions: a study of the adsorption kinetics of metal ions on ultrathin films for sensing applications $\dagger$}

\begin{abstract}
Monica Caselli*
The electrostatic multilayer self-assembly technique was employed to prepare fluorescent probes for mercury(॥) ions. Films showing an appreciable fluorescence emission were obtained by alternate adsorption of 5,10,15,20-tetrakis(4-sulfonatophenyl)porphyrin (TPPS) with polyelectrolytes. Fluorescence intensity quenching, arising from the coordinative interaction between the porphyrin and mercury ions, was monitored as a function of time. The response mechanism was studied on the basis of kinetic and equilibrium models for adsorption. The adsorption process of mercury ions on porphyrin-based films was described by the pseudo-second-order equation and the kinetic parameters were correlated with the initial concentrations of the metal ions in aqueous solution. The kinetic approach was evaluated as an alternative to monitoring the sensing responses at equilibrium.
\end{abstract}

\section{Introduction}

Fluorescent chemical sensors and probes have found wide applications in several fields, such as environmental monitoring, food and biochemical analysis, medical diagnosis. The advantages offered are high sensitivity of detection, low cost, "on-off" switchability, subnanometer spatial resolution with submicron visualization and submillisecond temporal resolution. ${ }^{\mathbf{1 , 2}}$

The awareness that mercury is a highly toxic global pollutant, characterized by accumulation and persistency properties in the environment and living organisms, ${ }^{3}$ has led to the use of selective analytical methods for determining mercury speciation, primary interests being addressed to the bioavailable chemical species. As regards the adverse effects of mercury on human health, the neurotoxicity of elemental and methylated forms and nephrotoxicity of "free" cation are well established. ${ }^{3}$ The United States Environmental Protection Agency (EPA) stated an upper limit of $2 \mu \mathrm{g} \mathrm{L}^{-1}$ for inorganic mercury in drinking water. ${ }^{4}$ The European Community included mercury among the priority substances for environmental quality standards (Directive 2013/39/EC) and fixed a more restrictive upper limit, the maximum allowable concentration of dissolved mercury in surface water being $0.07 \mu \mathrm{g} \mathrm{L}^{-1}$. The design of

Department of Chemical and Geological Sciences, University of Modena and Reggio Emilia, Via Campi 183, I-41125 Modena, Italy. E-mail: monica.caselli@unimore.it; Fax: +39059 373543; Tel: +390592055112

$\dagger$ Electronic supplementary information (ESI) available: Absorption and excitation spectra, AFM and microscope fluorescence images, linear fit to the kinetic pseudo-second-order equation. See DOI: 10.1039/c4ra09814e fluorescent sensors for mercury ions is the subject of several studies. However, they are usually developed in aqueous or organic solvents, ${ }^{5,6}$ though solid-state sensors immobilized on a solid surface offer important advantages from an applicative point of view. Furthermore, the interaction between the analyte and a surface differs from that in solution.

In this context, porphyrins possess spectroscopic and photophysical properties suitable for their use as fluorescent sensors, thanks to a large Stokes shift, high fluorescence quantum yield, good thermal and photochemical stability; the changes in spectroscopic and photophysical properties upon complexation of heavy metal ions, i.e. modification of the Soret absorption band as well as of the minor Q bands and a marked quenching of fluorescence emission, make them excellent building blocks for developing optical sensors for such analytes. As regards the use of these macrocycles for preparing solid-state optical sensing materials for heavy metal ions in aqueous solutions, a few examples were reported, both immobilized in polymeric membranes, ${ }^{7,8}$ sol-gel matrixes, ${ }^{9,10}$ and deposited as self-assembled films. ${ }^{\mathbf{1 1}, \mathbf{1 2}}$ The main limit in the use of porphyrins in solid-state sensors is the fluorescence quenching due to the formation of intermolecular weakly fluorescent aggregates, attributable to $\pi-\pi$ stacking interaction between two adjacent porphyrin cores, ${ }^{13}$ with a consequent reduction of sensitivity.

The electrostatic multilayer self-assembly technique has been rarely employed for sensing applications, in spite of the advantages offered by this approach over other traditional methods, such as the control of the film thickness and supramolecular structure through a clever modulation of many experimental variables. ${ }^{\mathbf{1 4 , 1 5}}$ This approach, based on the 
alternate adsorption of oppositely charged chemical species on a substrate, allows ultrathin film structures to be prepared; the thickness of each layer can be controlled in the range from 5 to $30 \AA .{ }^{\mathbf{1 6}}$ Electrostatically layer-by-layer self-assembly is a powerful method for developing fluorescent sensors, thanks to its versatility, based on the capability of selecting from a wide variety of charged fluorophores, together with properties of stability and reproducibility of multilayer films. Furthermore, multilayers can grow on many different substrates, such as planar slides, polymer microspheres, optical fibers, suggesting the interesting potentialities of electrostatic layer-by-layer selfassembly for a wide range of sensing applications. ${ }^{17,18}$

A crucial aspect concerning the development of sensing devices is represented by the adsorption process involved in analyte-solid probes interaction: a fast equilibration is usually assumed, disregarding the kinetic aspects, which, on the contrary, possess a fundamental importance when solid sensors or probes are employed. On the other hand, various kinetic models (pseudo-first-order, intraparticle diffusion, pseudosecond-order are the most widely used) have been proposed and discussed from a theoretical point of view; ${ }^{19-21}$ however, their application is generally addressed to bulk materials used as sorbents. ${ }^{22-24}$

In this paper, the development of electrostatically selfassembled multilayers (ESAM), prepared by alternate adsorption of 5,10,15,20-tetrakis(4-sulfonatophenyl)porphyrin (TPPS) with poly(diallyldimethylammonium chloride), able to act as efficient fluorescent probes for mercury(II) ions, is reported. Experimental parameters in the ESAM preparation were varied to improve the final sensing properties. We show how the electrostatically self-assembled technique can be employed in order to reduce the typical tendency of porphyrins to aggregation, through the choice of appropriate building blocks and experimental conditions of preparation. In order to test these films as fluorescent probes, changes in their spectroscopic and photophysical properties after dipping into solutions containing different concentrations of mercury(II) ions were characterized. Fluorescence emission quenching was observed for diluted solutions and solid-state ESAM films.

The adsorption process of mercury(II) ions on ESAM films used for sensing purposes was studied as a function of time, by monitoring fluorescence intensity changes, deriving from the interaction of TPPS with mercury ions. The response mechanism was investigated on the basis of various kinetic and equilibrium models for adsorption. It was shown how the kinetic parameters can be correlated with the initial mercury ion concentration in aqueous solution. Therefore, the analysis of the adsorption kinetics based on the study of fluorescence intensity quenching offers an alternative to equilibrium measurements, overcoming the problem of long equilibration times.

\section{Experimental}

\section{Materials}

5,10,15,20-tetrakis(4-sulfonatophenyl)porphyrin (TPPS) was purchased from Frontier Scientific; poly(diallyldimethylammonium chloride) (PDDA, Mw $=400000-500000)$ and poly(sodium-4styrenesulfonate) (PSS, Mw $\sim 1000000$ ) were obtained from Aldrich and used as received. Scheme 1 displays the chemical structures. All solutions were prepared using Milli-Q deionised water (resistivity about $18.2 \mathrm{M} \Omega \mathrm{cm}$ ); $\mathrm{pH}$ of TPPS solutions was adjusted by adding $1 \times 10^{-3} \mathrm{M}$ sodium hydroxide to obtain the free-base form. Glass microscope slides $(12.5 \times 45 \mathrm{~mm})$ were used as film substrates. They were treated under ultrasonication for 1 hour in an alkaline solution containing $5 \mathrm{~g}$ of $\mathrm{KOH}$ dissolved into a 1:8 water/2-propanol (Merck) mixture. Layer-by-layer selfassembled films were prepared using a PC-controlled self-coater (KSV model LM) by sequentially dipping the substrate into a $3.1 \times 10^{-2} \mathrm{M}(\mathrm{M}=$ moles of monomer per litre) solution of PDDA for 20 minutes, rinsing with deionised water, dipping into a $2 \times 10^{-4}$ M solution of TPPS in water at $\mathrm{pH} \sim 8$ ( $\mathrm{pH}$ was adjusted to avoid J-aggregation) for 20 minutes or, alternatively, into a $1.5 \times 10^{-2} \mathrm{M}$ PSS solution and final rinsing. Co-deposition of TPPS with PSS was obtained by dipping into solutions with different concentration ratios.

\section{Absorption and emission spectroscopy}

UV spectra were collected using a Varian Cary 100 Scan UV-vis spectrophotometer. Fluorescence measurements were performed on a Horiba Jobin Yvon Fluoromax-3 fluorometer. Spectra were corrected for the instrumental spectral sensitivity. Fluorescence quantum yields of films were measured using a solution of free-base TPPS in water at $\mathrm{pH}=8.0\left(\Phi_{\mathrm{F}}=0.056\right)^{25,26}$ placed in $1 \mathrm{~mm}$ path-length cell as the standard, by excitation at the Soret band. The absorbances of TPPS in ESAM and in solution were identical at the excitation wavelength. The corrections for refractive index were made, setting $n=1.45$, a typical value for similar organic films..$^{27,28,29}$ Reproducible positions of samples were ensured for all measurements.

A certified standard mercury(II) solution $\left(1 \mathrm{~g} \mathrm{~L}^{-1}, 2 \%\right.$ in nitric acid), purchased from CPI International, was diluted and acidified with $0.4 \%$ nitric acid in order to obtain a $1 \times 10^{-3} \mathrm{M}$ stock solution; working solutions were obtained by appropriate dilutions with borate buffer $(\mathrm{pH}=8.0)$. Mercury ion detection was performed by immersion of TPPS films in $1 \mathrm{~cm}$ path-length quartz cuvettes containing various metal ion solutions, whose concentrations ranged from $3.3 \times 10^{-5} \mathrm{M}$ to $3.3 \times 10^{-8} \mathrm{M}$; the corresponding absorption, excitation and emission fluorescence spectra were recorded at different time delays.

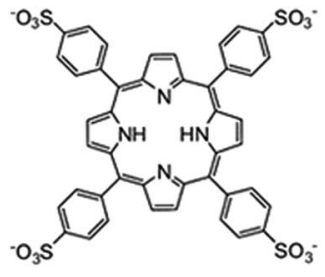

TPPS

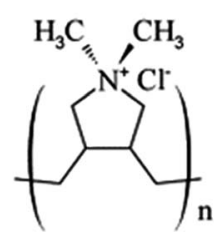

PDDA

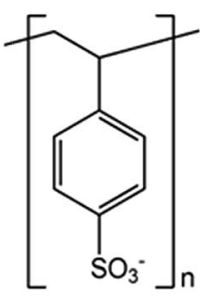

PSS 


\section{AFM and fluorescence microscopy}

The morphology of the film surfaces was studied using atomic force microscopy in tapping mode (CP Park Autoprobe). The surface analysis was performed with the PSI ProScan Image Processing software; the surface roughness was taken equal to the standard deviation of the $z$-value distribution within a $5 \times 5 \mu \mathrm{m}$ image area. Fluorescence microscopy images of the film surfaces were obtained with a confocal DM IRE2 Leica microscope equipped with a TCS SP2 scanning head and a laser source at $405 \mathrm{~nm}$.

\section{Adsorption kinetics and equilibrium}

All experiments were performed at $25{ }^{\circ} \mathrm{C}$. Adsorption kinetics of $\mathrm{Hg}$ (II) ions on PDDA/PSS/PDDA/TPPS ESAM was studied by monitoring fluorescence emission of TPPS. The surface coverage $\theta$ was determined as follows: the fluorescence intensity at time $t$, measured at $650 \mathrm{~nm}$, is given by two contributes, the emission of the free-base TPPS and the emission of the Hg-TPPS 1:1 complex. Considering the ratio $r$ between the fluorescence intensity of the unbound porphyrin at $650 \mathrm{~nm}$ and that of the complex in aqueous solution and assuming that such ratio does not change when the porphyrin is included in films, we obtain:

$$
I^{650}=(1-\theta) I_{0}^{650}+\theta \frac{I_{0}^{650}}{r}
$$

where $I_{0}^{650}$ and $I^{650}$ are, respectively, the fluorescence intensities measured at $650 \mathrm{~nm}$ before and after adding mercury ions.

The value of $r$, determined as an average of three measurements (see Fig. $\mathrm{S} 4 \dagger$ in ESI for representative spectra), is $6.0 \pm$ 0.6 .

Therefore, the determination of $\theta$ was performed using the expression:

$$
\theta=\frac{6\left(I_{0}^{650}-I^{650}\right)}{5 I_{0}^{650}}
$$

$\theta$ at time $t$ was the average value of at least three measurements. The kinetic data were examined using the pseudo-first-order, pseudo-second-order and intraparticle diffusion equations, according to the different functions of $\theta$ at time $t$ as a function of $t$. The surface coverage at equilibrium $\theta_{\mathrm{eq}}$ was derived from kinetic data and different equilibrium isotherm models were tested. In adsorption isotherms the equilibrium concentrations of mercury ions were obtained as follows: the TPPS total amounts (in mol) in ESAM were calculated from absorbance measurements and total surface of the films:

$$
\text { TPPS amount }(\mathrm{mol})=[\mathrm{TPPS}] V=\frac{A^{\max }}{\varepsilon^{\max } d} V=10^{-3} \frac{A^{\max }}{\varepsilon^{\max }} S,
$$

where $V$ is the film volume in $\mathrm{dm}^{3}, A$ is the maximum absorbance of free-base TPPS, $S$ is the film surface in $\mathrm{cm}^{2}, \varepsilon$ is the molar absorption coefficient at the $\mathrm{B}$ band maximum (466 $000 \mathrm{M}^{-1} \mathrm{~cm}^{-1}$ ), ${ }^{25} d$ is the film thickness in $\mathrm{cm}$.

The amount of $\mathrm{Hg}$ (II) adsorbed at time $t$ was derived by the $\theta$ value at that time; the equilibrium $\mathrm{Hg}$ (II) amounts and, hence, the equilibrium concentrations in solution were calculated by subtracting the adsorbed amount of $\mathrm{Hg}$ (II) from the initial amount in solution. The experimental kinetic and equilibrium data were fitted by means of a nonlinear least-squares algorithm.

\section{Results and discussion}

\section{Multilayer assembly of TPPS}

Absorption spectra of free-base TPPS in water $\left(c=3.9 \times 10^{-7} \mathrm{M}\right)$ and included in various ESAM are reported in Fig. 1. The absorption spectrum in aqueous solution $(\mathrm{pH}=8.0)$ is characterized by the main Soret (B) band at $413 \mathrm{~nm}$ with the associated vibronic band at $395 \mathrm{~nm}$ and four weak Q bands at 515, $552,580,633 \mathrm{~nm}$.

Such spectral features are attributable to TPPS in its freebase form $\left(\mathrm{p} K_{\mathrm{a}} \sim 4.7\right){ }^{30}$ the presence of $\mathrm{H}$ - or J-aggregates, whose formation in solution has been observed for diprotonated form, ${ }^{31,32}$ was not detected. In films the Soret band is redshifted with a maximum at about $420 \mathrm{~nm}$; this shift is intrinsic to the TPPS bound to PDDA. ${ }^{30}$ The shoulder at about $405 \mathrm{~nm}$, whose intensity decreases progressively by lowering the density of the porphyrin in the different films, is attributable to $\mathrm{H}$ aggregates. The fluorescence emission spectrum of free-base TPPS in solution, independent of the excitation wavelength chosen correspondingly to the Soret absorption, shows two main bands centred at about 645 and $705 \mathrm{~nm}$ (Fig. 2). The spectral characteristics are slightly altered in the films, where a red shift of the maxima to about $650 \mathrm{~nm}$ and $715 \mathrm{~nm}$ is observed; the fluorescence quantum yield is significantly reduced, as a function of the porphyrin density. Such results are in agreement with the observations that in thin solid films free-base TPPS can form H-aggregates, depending on the porphyrin concentration, whereas films obtained by the diacid form show a high content of both $\mathrm{H}$ - and J-aggregates. ${ }^{30}$ The general observation of fluorescence quenching in aggregated systems has been interpreted on the basis of exciton migration among porphyrin derivatives, followed by trapping at aggregate sites, characterized by much lower radiative decay rates. ${ }^{30}$

The excitation spectra, recorded at $\lambda_{\mathrm{em}}=655$ and $715 \mathrm{~nm}$, correspond to the absorption spectra, confirming that

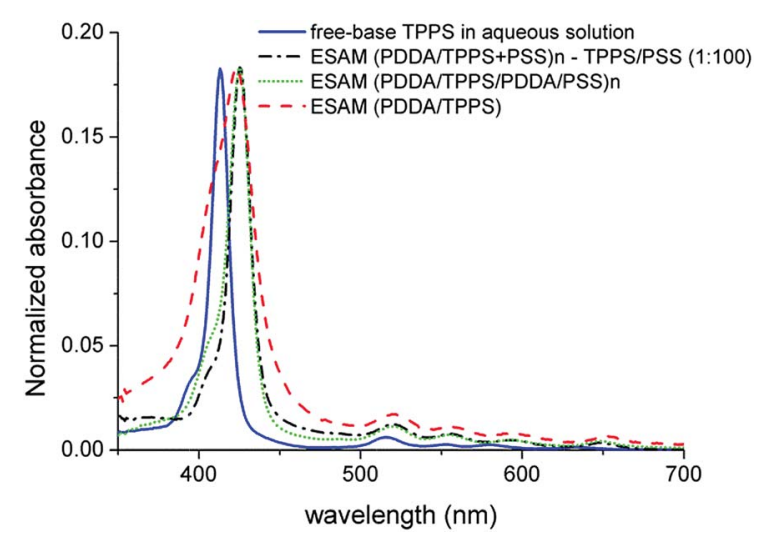

Fig. 1 Normalized absorption spectra of TPPS in aqueous solution $(\mathrm{pH}=8.0)$ and in different multilayers. 


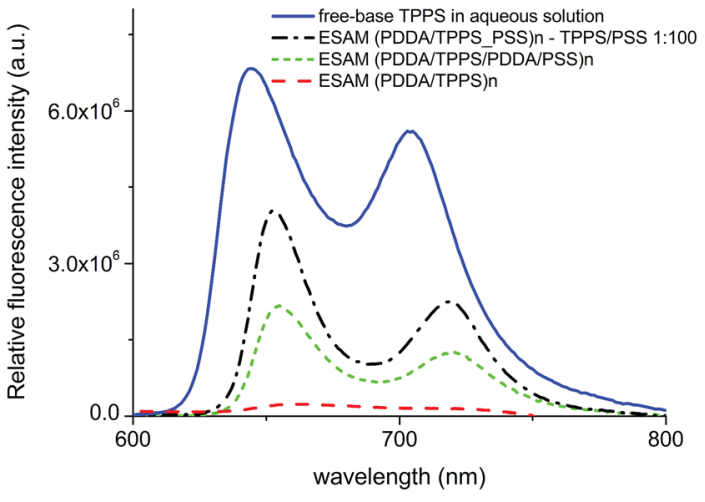

Fig. 2 Relative fluorescence emission of TPPS in aqueous solution $(\mathrm{pH}=8.0)$ and in different multilayers. $\lambda_{\mathrm{ex}}=421 \mathrm{~nm}$.

absorption and emission belong to the same main fluorophore; the large shoulder at about $405 \mathrm{~nm}$ results less intense than in absorption, in accordance with the assignment to weakly fluorescent aggregates (Fig. S1 in ESI $\dagger$ ).

The observations deduced from absorption and relative fluorescence emissions indicate that the tendency to form $\mathrm{H}$-aggregates is important in (PDDA/TPPS $)_{n}$ ESAM and is reduced by the introduction of PSS: by alternating each PDDA/TPPS bilayer with a PDDA/PSS bilayer, yielding a (PDDA/PSS/PDDA $\mathrm{TPPS}_{n}$ multilayer, or co-depositing TPPS with PSS, yielding a $(\text { PDDA/TPPS-PSS })_{n}$ multilayer, we are able to obtain films with a significant fluorescent emission, by tuning the experimental conditions of preparation. Progressively higher values of fluorescence quantum yields (Table 1) were obtained in films where the TPPS was co-deposited with PSS, increasing the mixing ratio PSS : TPPS in the dipping solution from $1: 1$ to $100: 1$; the values of fluorescence quantum yield do not significantly vary as a function of the number of layers. Such observations confirm that de-aggregation, with consequent enhancement of the fluorescence emission, can be obtained by means of two different strategies, ${ }^{33}$ which make it possible to separate the porphyrin fluorophores and reduce the $\pi$-stacking aggregation.

In both cases, multilayers grow regularly (Fig. S2 in ESI $\dagger$ ). A higher amount of TPPS per layer is deposited in alternate (PDDA/TPPS/PDDA/PSS) ${ }_{n}$ films (a) with respect to films in which TPPS is co-deposited with PSS in 1:100 ratio (b). In codeposited films, the amount of TPPS adsorbed is a function of its concentration in the dipping solution.

Table 1 Fluorescence quantum yields (average values) of different types of ESAM

\begin{tabular}{ll}
\hline Film & $\Phi_{\mathrm{F}}$ \\
\hline$(\text { PDDA/PSS/PDDA/TPPS })_{n}$ & $0.011 \pm 0.002$ \\
$(\text { PDDA/TPPS-PSS } 1: 100)_{n}$ & $0.030 \pm 0.005$ \\
$(\text { PDDA/TPPS-PSS } 1: 20)_{n}$ & $0.022 \pm 0.004$ \\
$(\text { PDDA/TPPS-PSS } 1: 10)_{n}$ & $0.011 \pm 0.002$ \\
$(\text { PDDA/TPPS-PSS } 1: 5)_{n}$ & $0.003 \pm 0.001$ \\
$(\text { PDDA/TPPS-PSS } 1: 1)_{n}$ & $0.001 \pm 0.0005$
\end{tabular}

A balance between a good fluorescence quantum yield and an appreciable amount of TPPS was obtained in ESAM (PDDA/PSS/ PDDA/TPPS $)_{n}$ where PSS and TPPS were alternated as negatively charged building blocks. For such kind of films, $n$ was varied from 1 to 9: the values of fluorescence quantum yield confirmed a substantial independency of the number of layers.

\section{Sensing properties of multilayers}

The preliminary measurements were performed by adding different volumes of a $1 \times 10^{-4} \mathrm{M}$ mercury(II) ion solution to $\sim 10^{-6}$ to $10^{-7} \mathrm{M}$ TPPS solutions in borate buffer $(\mathrm{pH}=8.0)$, to obtain final concentrations of mercury ranging from $6.7 \times 10^{-8}$ to $4.7 \times 10^{-6} \mathrm{M}$ : marked changes both in absorption and emission spectra were observed. The coordinative interaction between TPPS and mercury induces a red-shift of the Soret (from 413 to $420 \mathrm{~nm}$ ) and the Q bands, whose number results reduced as a consequence of the increased symmetry ${ }^{34}$ (Fig. S3 in ESI $\dagger$ ). The emission spectrum of TPPS in solution is characterized by two bands at 645 and $705 \mathrm{~nm}$; upon addition of mercury ions, a new band at $605 \mathrm{~nm}$ progressively increases and the maximum shifts to $656 \mathrm{~nm}$ (Fig. S4 in ESI†). These results are in agreement with previous studies and indicate the formation of a $1: 1 \mathrm{Hg}$-TPPS complex. ${ }^{26,35}$ It has been established that mercury(II) in aqueous solution at $\mathrm{pH}=8$ forms predominantly hydroxo complexes, ${ }^{25}$ which can form, upon interaction with TPPS, Hg-TPPS complexes. In the present work, $\mathrm{OH}^{-}$ligands are not mentioned and we refer to mercury(II) ions.

The interaction of TPPS included in ESAM with mercury(II) ions does not substantially change the position of the Soret absorption band ( $422 \mathrm{~nm}$ ), already red-shifted by the interaction with PDDA, whereas the only detectable $\mathrm{Q}$ band is centred at about $556 \mathrm{~nm}$ (Fig. 3). In emission spectra two main bands are present at 605 and $650 \mathrm{~nm}$ (see Fig. 4), according to the formation of a $1: 1 \mathrm{Hg}$-TPPS complex. Excitation spectra, recorded at $\lambda_{\mathrm{em}}=655 \mathrm{~nm}$, reproduce the absorption spectrum of the residual TPPS, whereas the excitation spectra at $\lambda_{\mathrm{em}}=605 \mathrm{~nm}$ shows a new excitation band at about $430 \mathrm{~nm}$, suggesting a red-shift of the Soret absorption band of TPPS included in ESAM upon

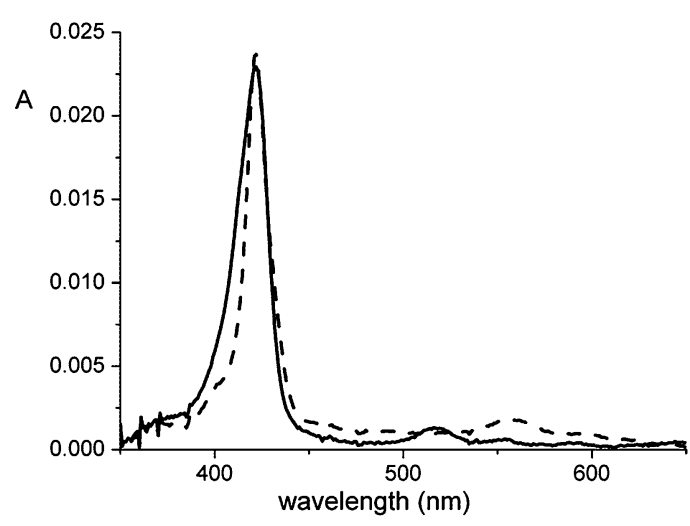

Fig. 3 Absorption spectra of PDDA/PSS/PDDA/TPPS ESAM before (continuous line) and after (dashed line) dipping in a $3.3 \times 10^{-5} \mathrm{M}$ solution of $\mathrm{Hg}(I)$ ions. 


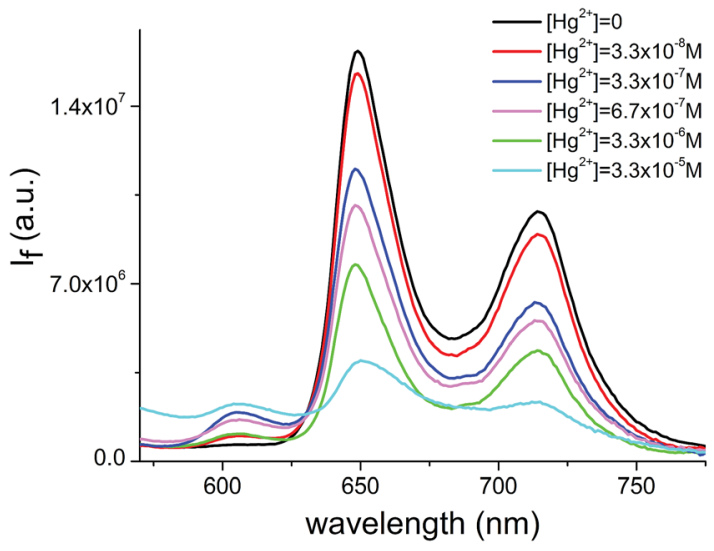

Fig. 4 Fluorescence emission quenching of TPPS in PDDA/PSS/ PDDA/TPPS ESAM upon interaction with mercury(I) ions (concentration range from $3.3 \times 10^{-5}$ to $3.3 \times 10^{-8} \mathrm{M}$ ) and rise of the emission at $605 \mathrm{~nm}$. Initial fluorescence emissions were normalized. Dipping time $=60 \mathrm{~min}$

coordination by mercury. Although the formation of $2: 2$ and $3: 2$ non-fluorescent complexes cannot be completely excluded, features of both absorption and emission spectra indicate the prevalent formation of a $\mathrm{Hg}$-TPPS complex in $1: 1$ ratio. $^{26,35}$

The most significant observation is a marked quenching of the fluorescence of TPPS contained in ESAM, whose extent depends on the mercury concentration. The response time is correlated with the number of layers: films formed by a high number of layers require excessively long time for equilibration, likely because of slow diffusion of the analyte within the film. (PDDA/PSS/PDDA/PSS $)_{n}$ films with $n=1,2$ show the best properties as sensing materials, since they combine acceptable response time (less than one hour) with the above mentioned properties of good fluorescence quantum yields and optical density. Fig. 4 shows the fluorescence quenching of TPPS included in PDDA/PSS/PDDA/TPPS films as a function of mercury(II) ion concentration in the range $3.3 \times 10^{-8}$ to $3.3 \times 10^{-5}$ M. The fluorescent probe may find application to detect mercury in such concentration range. For lower concentration the fluorescence quenching was not detectable.

TPPS-based films display changes in fluorescence intensity as a consequence of the existence of TPPS in two different forms (free and complexed), one of which is characterized by a reduced emission quantum yield. Coordination of the porphyrin ring by mercury(II) ions yields out-of-plane complexes, in which the metal ion is located above the plane of the pyrrole ring, which results distorted, as a consequence of a metal centre radius exceeding the critical value of $80 \mathrm{pm} .^{26,35}$ Out-of-plane metalloporphyrins are characterized by different spectral and photophysical properties with respect to those of more common in-plane metalloporphyrins, since the coordination of a metal induces a red-shift of the Soret band and a marked reduction of the fluorescence quantum yield. The latter observation has often been interpreted on the basis of the "heavy atom" effect, which induces a more efficient deactivation to the triplet state. ${ }^{11}$ Recent studies, ${ }^{26}$ however, suggest that this is not the dominant factor and, on the contrary, an important role can be attributed to the deformation of the structure and reduction of symmetry associated with the out-of-plane coordination, which favours the internal conversion deactivation with respect to the radiative path.

The selectivity of TPPS-based films for mercury ions in aqueous solution was investigated. The fluorescence response to various transition-metal cations $\left(\mathrm{Pb}^{2+}, \mathrm{Cd}^{2+}, \mathrm{Cu}^{2+}, \mathrm{Zn}^{2+}\right)$ and some Group II $\left(\mathrm{Ca}^{2+}\right.$ and $\left.\mathrm{Mg}^{2+}\right)$ was evaluated in comparison with $\mathrm{Hg}^{2+}$. Fluorescence emission of TPPS remains unaffected in solutions of $\mathrm{Ca}^{2+}$ and $\mathrm{Mg}^{2+}$. Whereas mercury ions induce a marked quenching of fluorescence intensity, the other transition metal ions give rise to a less degree of quenching. $\mathrm{Zn}^{2+}$, which was found to interfere to other related systems, ${ }^{9}$ produces only minor changes in fluorescence emission of TPPS layer. Divalent copper ions appear the most interfering species. Such observations can be interpreted on the basis of different spectroscopic properties of metalloporphyrins and their formation constants. Out-of-plane metalloporphyrins, formed by ions that possess a radius higher than the critical values, show similar spectroscopic properties: $\mathrm{Cd}^{2+}$ and $\mathrm{Pb}^{2+}$ may interfere in fluorimetric determination of $\mathrm{Hg}^{2+}$, because form with TPPS out-ofplane complexes, characterized by similar properties in absorption and emission. ${ }^{36}$ On the contrary, the in-plane metalloporhyrins have different spectral characteristics. The most interfering ion, $\mathrm{Cu}^{2+}$, although possesses a radius around the critical value, forms with TPPS a in-plane complex, characterized by spectroscopic properties similar to those of the freebase. The excitation at $420 \mathrm{~nm}$, correspondently to the Soret band of TPPS in free-base form, already red-shifted in films, may be not selective for ions that forms out-of-plane complexes and for $\mathrm{Cu}^{2+}$. As regards the equilibrium formation constants, out-of-plane metalloporphyrins in general show higher values than the in-plane ones. ${ }^{36} \mathrm{Zn}^{2+}$ forms a border-line complex, characterized by absorption properties similar to those of outof-plane complexes; its minor interference can be explained on the basis of a higher fluorescence quantum yield and a lower formation constant with respect to the out-of-plane metalloporphyrins. Though such feature has to be deepened, we suggest that the detection of mercury ions in aqueous solutions containing interfering cations, such as $\mathrm{Cu}^{2+}, \mathrm{Pb}^{2+}, \mathrm{Cd}^{2+}$, can be performed by studying the different kinetics of interaction. ${ }^{36}$

\section{AFM and fluorescence microscopy characterization}

The comparison among various types of ESAM films, performed by AFM, showed that they are characterized by different surface morphology, depending on the nature and the number of layers. It appears quite smooth and uniform in PDDA/PSS/PDDA/TPPS film: the estimated surface roughness (root-mean-square roughness, $R_{\mathrm{ms}}$ ), calculated within an area of $5 \times 5 \mu \mathrm{m}$, (Fig. 5) was $1.0 \mathrm{~nm}$. In PDDA/TPPS films the morphological heterogeneity increases (Fig. S5 in ESI $\dagger$ ), the $R_{\mathrm{ms}}$ value being $2.5 \mathrm{~nm}$. Such observations are consistent with the significant tendency to form $\mathrm{H}$-aggregates in (PDDA/TPPS $)_{n}$ ESAM, which is reduced by the introduction of PSS, as pointed out by both absorption and emission spectroscopic measurements. 

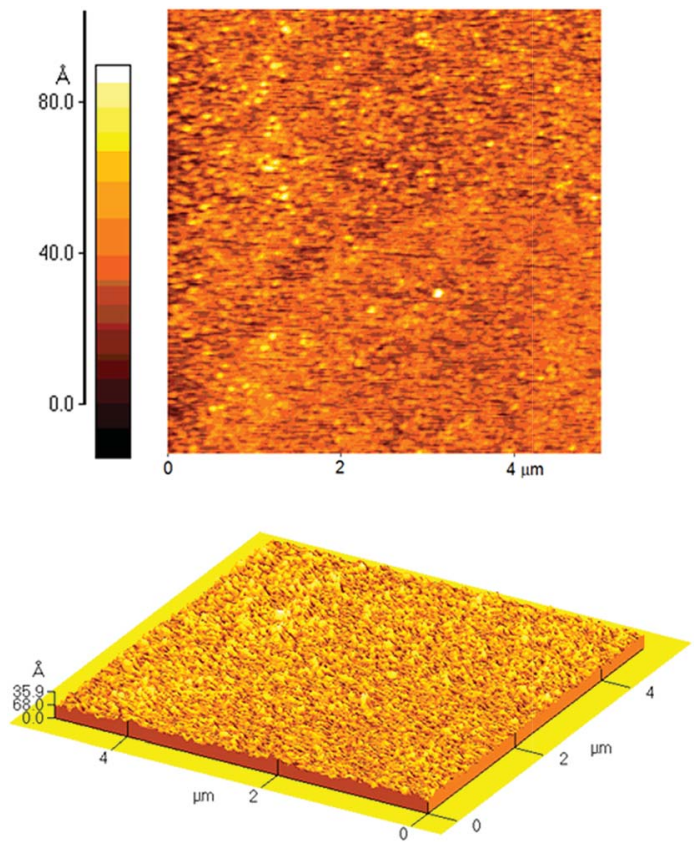

Fig. 5 AFM image $(5 \times 5 \mu \mathrm{m})$ of a PDDA/PSS/PDDA/TPPS film (top) and the corresponding three-dimensional representation (bottom).

Literature data report that AFM experiments on films consisting of different numbers of PDDA/ $\mathrm{H}_{4}$ TPPS $^{2-}$ bilayers (where TPPS is present in diacid form) exhibited a regular increase in roughness, because of aggregates formation. ${ }^{29}$ On the basis of such results, we can confirm, according to fluorescence measurements, the low grade of aggregation in PDDA/PSS/PDDA/TPPS films, where porphyrin is mainly present as monomer.

Fluorescence microscopy investigation showed that the monomer emission was fairly homogeneous with brighter spots distributed not homogeneously in the film (Fig. S6 in ESI $\dagger$ ). The emission spectra acquired in five different regions of interest (ROI) confirmed the presence of TPPS.

\section{Adsorption kinetics and equilibrium}

The features of the adsorption equilibria have been widely investigated on a theoretical basis, whereas the kinetic description of the adsorption processes, due to its complexity, remained essentially empirical for many years. Adsorption process of a chemical species on a solid surface can be described by four main consecutive steps: transport in the bulk solution, diffusion across the film surrounding the solid surface, diffusion in the pores of the sorbent (intraparticle diffusion) and surface reaction. ${ }^{19}$ The overall adsorption rate is controlled by the slowest among these steps. Several kinetic models can be used to describe the rate of adsorption at solid/ liquid interface: Langmuir, ${ }^{37}$ statistical rate theory (SRT), pseudo-first-order (Lagergren equation), pseudo-second-order, Elovich $^{19}$ and intraparticle diffusion model ${ }^{38}$ can be recognized as the most commonly employed. Two main groups can be identified: (a) models assuming that the diffusion of the sorbate in the pores of the sorbent controls the rate of adsorption, and (b) models assuming that the rate of adsorption is governed by the rate of the surface reaction. ${ }^{21}$

Recently, a model for the description of mixed surface reaction and diffusion-controlled adsorption kinetics was proposed. ${ }^{39}$ The Langmuir kinetic model, leading at equilibrium to Langmuir adsorption isotherm, considers the case of an ideal, energetically homogeneous solid surface and one-site occupancy adsorption model. The pseudo-first-order (eqn (1) and (1b)) and pseudosecond-order (eqn (2) and (2b)) equations were at first proposed on empirical bases; they are the most commonly used expressions to describe adsorption kinetics data, concerning radically different kinds of sorbents and solutes..$^{22,23,40,41}$

The expression of the integrated pseudo-first-order kinetic equation is given by.

$$
\theta_{(t)}=\theta_{(\mathrm{eq})}\left(1-e^{k_{1} t}\right)
$$

where $\theta_{(\mathrm{t})}$ and $\theta_{(\mathrm{eq})}$ are the fraction of surface covered at time $t$ and at equilibrium, $k_{1}$ is the pseudo-first-order rate coefficient.

Eqn (1) can be written in linear form:

$$
\ln \left(\theta_{(\mathrm{eq})}-\theta_{(\mathrm{t})}\right)=\ln \theta_{(\mathrm{eq})}-k_{1} t
$$

The expression of the integrated pseudo-second-order kinetic equation is given by

$$
\theta_{(t)}=\frac{k_{2} N_{(\mathrm{eq})} \theta_{(\mathrm{eq})} t}{1+k_{2} N_{(\mathrm{eq})} t}
$$

where $N_{(\mathrm{eq})}$ is the amount of solute per unit mass of adsorbent adsorbed at equilibrium, $k_{2}$ is the pseudo-second-order rate coefficient. A linear representation of this equation is given by

$$
\frac{t}{\theta_{(t)}}=\frac{1}{\theta_{(e q)}} t+\frac{1}{k_{2} N_{(e q)} \theta_{(e q)}}
$$

Different theoretical interpretations of these equations have been proposed, initially adopting the classical Langmuir model of adsorption kinetics, ${ }^{\mathbf{2 0 , 4 2}}$ where the adsorption is seen as a chemical reaction on a solid surface: the pseudo-first-order and pseudo-second-order equations are special cases of the Langmuir rate equation, obtained for high and low initial concentrations of the solute, respectively. The approach based on the SRT (statistical rate theory of interfacial transport) theory derives the pseudo-first-order and pseudo-second-order equations as simplified forms of a general kinetic equation, developed for a monolayer, one-site-occupancy model of adsorption on both homogeneous and heterogeneous solid surfaces, assuming that the process is controlled by the rate of surface reactions. ${ }^{43,44}$ The choice of extending the model by including strongly heterogeneous surfaces was due to the observed good applicability of the two equations to many real systems. According to this interpretation, both the pseudo-first-order and the pseudo-second-order kinetic equations are simplified forms of a more general expression under some definite assumptions: it appears that the former is generally applicable for the range of longer adsorption times, not far from the equilibrium conditions. 
In order to study the adsorption kinetics, the time evolution of the fractional surface coverage on PDDA/PSS/PDDA/TPPS ESAM, obtained from the fluorescence intensity quenching at $\mathrm{pH}=8$ after dipping in aqueous mercury(II) ion solutions with different concentrations, was investigated: it is reported in Fig. 6, together with the curves resulting from a fit to the pseudo-first-order and pseudo-second-order equations. The kinetic study was performed only on ESAM containing a single layer of TPPS; progressively longer times of adsorption were observed in films characterized by a higher number of layer. The analysis was performed in a concentration range $3.3 \times 10^{-5}$ to $3.3 \times 10^{-8}$. The kinetic behaviour of $3.3 \times 10^{-8} \mathrm{M}$ mercury solution is not reported, since the change of fluorescence intensity was detectable only at initial times (less than 10 minutes).

The adsorption process was found to be dependent on the initial concentration of mercury ions $\left(\left[\mathrm{Hg}^{2+}\right]_{0}\right)$ : higher concentrations required longer equilibration times. The pseudo-secondorder equation provides a good fit of all experimental data, both at short and longer times, approaching equilibrium (Table 2). On the contrary, the pseudo-first-order equation is unable to describe the kinetic data over the whole temporal range. The surface coverage at equilibrium, not directly determinable with sufficient accuracy, owing to long equilibration times, was obtained. As concentration of mercury increases, the adsorption process is characterized by higher values of both $\theta_{\text {eq }}$ and $k_{2}$. For an initial concentration $3.3 \times 10^{-5} \mathrm{M}$, a saturation of the adsorption sites $\left(\theta_{\text {eq }} \sim 1\right)$ is reached at equilibrium. The pseudosecond-order linear representation (eqn (2b)), reported in Fig. S7 in ESI, $\uparrow$ provides the slopes and intercept values, both dependent on the initial concentration of the analyte (Table S1 in ESI $\dagger$ ).

Therefore, the kinetic approach offers the possibility of correlating the fluorescence observables with mercury ion concentrations on a shorter time scale and higher accuracy than applies to equilibrium measurements. This observation is of

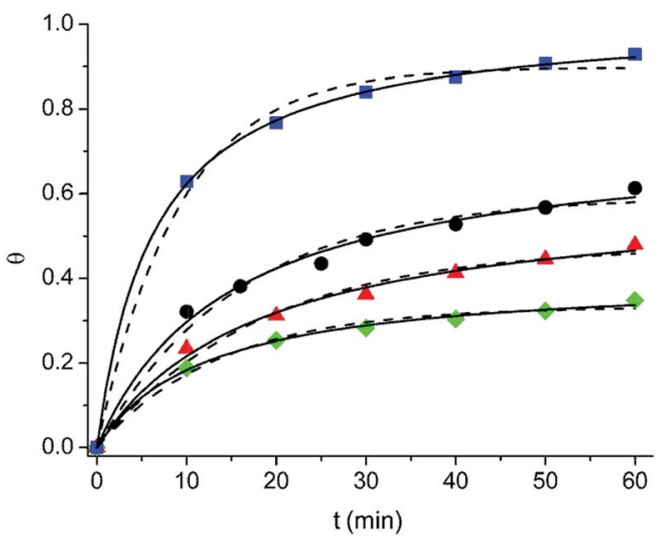

Fig. 6 Adsorption kinetics of mercury(॥) ions, at four different initial concentrations, on PDDA/PSS/PDDA/TPPS ESAM at $25^{\circ} \mathrm{C}$ in borate buffer at $\mathrm{pH}=8.0$ : best fit curves resulting from nonlinear leastsquares fitting of experimental data are reported (solid line, pseudosecond-order equation; dashed line, pseudo-first-order equation). The initial mercury(॥) ion concentrations were: $3.3 \times 10^{-5} \mathrm{M}(\boldsymbol{\square}), 3.3 \times$ $10^{-6} \mathrm{M}(\bullet), 6.7 \times 10^{-7} \mathrm{M}(\mathbf{\Delta}), 3.3 \times 10^{-7} \mathrm{M}(\bullet)$.
Table 2 Pseudo-first-order and pseudo-second-order kinetic parameters for mercury(॥) ion adsorption on PDDA/PSS/PDDA/TPPS ESAM in borate buffer at $\mathrm{pH}=8.0$, obtained from the nonlinear leastsquares fitting of experimental data to eqn (1) and (2). Adjusted $R^{2}$ values are reported

Pseudo-first-order model

\begin{tabular}{llll}
\hline$\left[\mathrm{Hg}^{2+}\right]_{0}\left(\mathrm{~mol} \mathrm{~L}^{-1}\right)$ & $\theta_{(\mathrm{eq})}$ & $k_{1}\left(\mathrm{~min}^{-1}\right)$ & $R_{\mathrm{adj}}{ }^{2}$ \\
\hline $3.3 \times 10^{-5}$ & 0.90 & 0.1100 & 0.994 \\
$3.3 \times 10^{-6}$ & 0.59 & 0.0634 & 0.982 \\
$6.7 \times 10^{-7}$ & 0.48 & 0.0555 & 0.986 \\
$3.3 \times 10^{-7}$ & 0.33 & 0.0736 & 0.989 \\
\hline
\end{tabular}

Pseudo-second-order model

\begin{tabular}{lllll}
\hline$\left[\mathrm{Hg}^{2+}\right]_{\mathrm{o}}\left(\mathrm{mol} \mathrm{L}^{-1}\right)$ & $k_{2} N_{(\mathrm{eq})} \theta_{(\mathrm{eq})}\left(\mathrm{min}^{-1}\right)$ & $k_{2} N_{(\mathrm{eq})}\left(\mathrm{min}^{-1}\right)$ & $\theta_{(\mathrm{eq})}$ & $R_{\mathrm{adj}}{ }^{2}$ \\
\hline $3.3 \times 10^{-5}$ & 0.1599 & 0.1567 & 1.02 & 0.999 \\
$3.3 \times 10^{-6}$ & 0.0498 & 0.0675 & 0.74 & 0.993 \\
$6.7 \times 10^{-7}$ & 0.0338 & 0.0558 & 0.60 & 0.995 \\
$3.3 \times 10^{-7}$ & 0.0336 & 0.0832 & 0.40 & 0.997
\end{tabular}

fundamental importance in the prospect of using ESAM including a single layer of porphyrin as probes to detect the mercury ion concentration in water. The kinetic approach can be evaluated as an alternative to the usual study of the sensing response at equilibrium: the relationship proposed ${ }^{\mathbf{3 4 , 4 5}}$ for a fluorescent probe that exists in solution in two states (free and bound to the analyte), although applied also to solid-state sensors, ${ }^{7,8,12}$ is subject to the important limitation of long equilibration times.

The theoretical interpretation of the pseudo-second-order equation $^{\mathbf{4 3 4 4}}$ is generally based on the assumption of an adsorption process at solid/solution interface controlled by the surface reaction. In the region of short adsorption times, the diffusional model predicts the square-root dependence of the surface coverage on time. ${ }^{21}$ The linear fit to the pseudo-secondorder equation over the whole temporal range (Fig. S7 in ESI $\dagger$ ) suggests that the diffusion does not control the process: in particular, in a mixed surface reaction and diffusion-controlled kinetic model, a deviation from linearity is observed. ${ }^{39}$ Even if we can not rule out that intraparticle diffusion may play a role at initial times, the nature of the ESAM studied, i.e. films including a single layer of TPPS, allows us to suggest that the adsorption kinetics of mercury ions is controlled by the complexation reaction with TPPS. We can suppose that the surface reaction represents the limiting step, whereas intraparticle diffusion possibly acquires importance as the number of layers increases. The room temperature $\left(25^{\circ} \mathrm{C}\right)$ equilibrium isotherms for the adsorption of mercury(II) ions on PDDA/PSS/ PDDA/TPPS ESAM were tested using the models of Langmuir (eqn (3)), and Langmuir-Freundlich (eqn (4), also known as Sips). ${ }^{46}$

$$
\theta_{\text {eq }}=\frac{K_{\mathrm{L}} c_{\mathrm{eq}}}{1+K_{\mathrm{L}} c_{\mathrm{eq}}}
$$




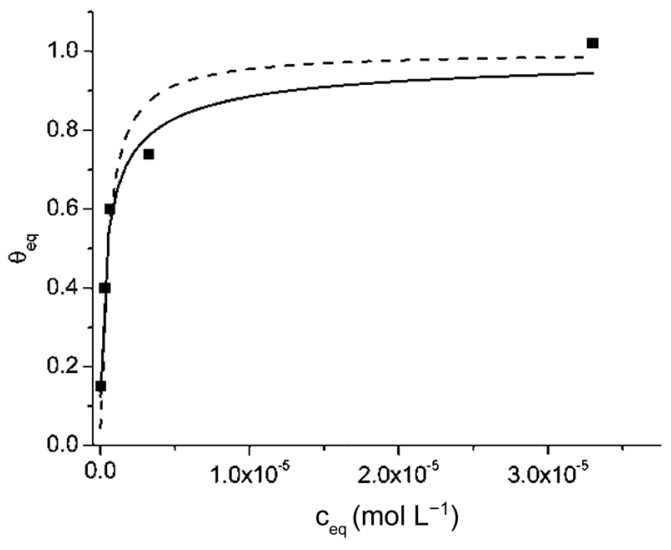

Fig. 7 Adsorption isotherm at $25^{\circ} \mathrm{C}$ of mercury(॥) ions on PDDA/PSS/ PDDA/TPPS ESAM in borate buffer at $\mathrm{pH}=8.0$ and nonlinear best least squares fits to Langmuir-Freundlich (solid line) and Langmuir (dashed line) models. Adjusted $R^{2}$ value are 0.97 and 0.93

where $K_{\mathrm{L}}$ is the Langmuir adsorption constant and $c_{\mathrm{eq}}$ the equilibrium sorbate concentration

$$
\theta_{\mathrm{eq}}=\frac{\left(K_{\mathrm{LF}} c_{\mathrm{eq}}\right)^{n}}{1+\left(K_{\mathrm{LF}} c_{\mathrm{eq}}\right)^{n}}
$$

where $K_{\mathrm{LF}}$ is the Langmuir-Freundlich adsorption constant, $n$ a heterogeneity factor and $c_{\text {eq }}$ the equilibrium sorbate concentration. The curves calculated with the best fit parameters obtained by applying the Langmuir and Langmuir-Freundlich model, which considers energetically heterogeneous adsorption sites, are shown in Fig. 7. From the graphical evaluation, the experimental data are well described by the LangmuirFreundlich model, except for the highest surface coverage, whose value, obtained from the pseudo-second-order fit, is overestimated. The best fit parameters give a value of the affinity constant $K_{\mathrm{LF}}=2.4 \times 10^{6}\left(\mathrm{~mol} \mathrm{~L}^{-1}\right)$ and a heterogeneous parameter $n=0.65$.

On the basis of these results, we can suggest that our films, chemically homogeneous, offer a one-site-occupancy adsorption on an energetically heterogeneous lattice of adsorption sites, as a consequence of a morphological, although not marked, heterogeneity, as shown by the AFM analyses. Such observation is consistent with a one-step adsorption process, recognizable, on the basis of the absorption and emission spectroscopic evidences, as the complexation between TPPS contained in ESAM films and mercury(II) ions.

\section{Conclusions}

The electrostatic multilayer self-assembly technique was employed to prepare porphyrin-based fluorescent probes able to detect mercury(II) ions in aqueous solution. PDDA/PSS/PDDA/ TPSS films showed the best properties as sensing materials, in terms of response time and fluorescence emission properties. The deposition of a layer of PSS reduced the formation of weakly fluorescent aggregates between adjacent porphyrins, resulting in films characterized by a higher fluorescence quantum yield.
The fluorescence emission of TPPS contained in such ultrathin solid films was efficiently quenched upon interaction with mercury ions in aqueous solutions, due to the formation of a $\mathrm{Hg}$-TPPS complex in 1:1 ratio. The adsorption process was studied by monitoring the fluorescence intensity as a function of time. The pseudo-second-order equation provided a good fit of all experimental data, both at short and longer times. We suggested that the adsorption kinetics of mercury ions is controlled by the complexation reaction. It was shown how the kinetic parameters can be correlated with the initial concentration of mercury ions, providing the basis for the use of these porphyrin-based ESAM as probes for the detection of mercury ions. The study of the extent of fluorescence intensity quenching as a function of time offers an alternative to equilibrium measurements, overcoming the problem of a slow equilibration, typical of solid-state probes.

\section{Acknowledgements}

This work was partially funded by grant 2006039379_001 (PRIN 2006) from MIUR (Ministero dell'Istruzione, Università e Ricerca).The "Centro Interdipartimentale Grandi Strumenti" of the University of Modena and Reggio Emilia is kindly acknowledged for AFM and fluorescence microscopy measurements.

\section{Notes and references}

1 A. P. de Silva, H. Q. N. Gunaratne, T. Gunnlaugsson, A. J. M. Huxley, C. P. McCoy, J. T. Rademacher and T. E. Rice, Chem. Rev., 1997, 97, 1515-1566.

2 J. F. Callan, A. P. de Silva and D. C. Magri, Tetrahedron, 2005, 61, 8551-8588.

3 L. Magos, Mercury and its Effects on Environment and Biology, in Metal Ions in Biological Systems, ed. A. Sigel and H. Sigel, Dekker, New York, 1997, vol. 34, ch. 11.

4 USEPA. EPA, report 815-B-09-002: EPA protocol for the second review of the existing national primary drinking water regulations (update), Office of Water, EPA, Washington, 2009.

5 D. Svechkarev, B. Dereka and A. Doroshenko, J. Phys. Chem. A, 2011, 115, 4223-4230.

6 V. Balla, R. Tejpal, M. Kumar and A. Sethi, Inorg. Chem., 2009, 48, 11677-11684.

7 W. H. Chan, R. H. Yang and K. M. Wang, Anal. Chim. Acta, 2001, 444, 261-269.

8 Y. Yang, J. Jiang, G. Shen and R. Yu, Anal. Chim. Acta, 2009, 636, 83-88.

9 M. Plaschke, R. Czolk and H. J. Ache, Anal. Chim. Acta, 1995, 304, 107-113.

10 D. Delmarre, R. Méallet, C. Bied-Charreton and R. B. Pansu, J. Photochem. Photobiol., A, 1999, 124, 23-28.

11 L. S. Dolci, E. Marzocchi, M. Montalti, L. Prodi, D. Monti, C. Di Natale, A. D'Amico and R. Paolesse, Biosens. Bioelectron., 2006, 22, 399-404.

12 Z. Fang and B. Liu, Tetrahedron Lett., 2008, 49, 2311-2315. 
13 C. A. Hunter and J. K. M. Sanders, J. Am. Chem. Soc., 1990, 112, 5525-5534.

14 G. Decher, Science, 1997, 277, 1232-1237.

15 Multilayer Thin Films, ed. G. Decher and J. B. Schlenoff, Wiley-VCH, New York, 2003.

16 G. Decher, Y. Lvov and J. Schmitt, Thin Solid Films, 1994, 244, 772-777.

17 P. S. Grant and M. J. McShane, IEEE Sens. J., 2003, 3, 139146.

18 S.-H. Lee, J. Kumar and S. K. Tripathy, Langmuir, 2000, 16, 10482-10489.

19 W. Plazinski, W. Rudzinski and A. Plazinska, Adv. Colloid Interface Sci., 2009, 152, 2-13.

20 S. Azizian, J. Colloid Interface Sci., 2004, 276, 47-52.

21 W. Rudzinski and W. Plazinski, J. Phys. Chem. C, 2007, 111, 15100-15110.

22 S. S. Gupta and K. G. Bhattacharyya, Adv. Colloid Interface Sci., 2011, 162, 39-58.

23 Y.-S. Ho, J. Hazard. Mater., 2006, B136, 681-689.

24 W. Plazinski, Adv. Colloid Interface Sci., 2013, 197-198, 5867.

25 O. Horvát, Z. Valicsek and A. Vogler, Inorg. Chem. Commun., 2004, 7, 854-857.

26 Z. Valicsek, G. Lendvay and O. Horvát, J. Phys. Chem. B, 2008, 112, 14509-14524.

27 J. Frueh, G. Reiter, H. Mohwald, Q. He and R. Krastev, Phys. Chem. Chem. Phys., 2013, 15, 483-488.

28 F. J. Arregui, B. Dickerson, R. O. Klaus, I. R. Matias and K. L. Cooper, IEEE Photonics Technol. Lett., 2001, 13, 13191321.

29 E. Collini, C. Ferrante, R. Bozio, A. Lodi and G. Ponterini, J. Mater. Chem., 2006, 16, 1573-1578.
30 P. G. Van Patten, A. P. Shreve and R. J. Donohoe, J. Phys. Chem. B, 2000, 104, 5986-5992.

31 D. L. Akins, H.-R. Zhu and C. Guo, J. Phys. Chem., 1994, 98, 3612-3618.

32 D. L. Akins, S. Özçelik, H.-R. Zhu and C. Guo, J. Phys. Chem., 1996, 100, 14390-14396.

33 A. Lodi, M. Caselli, B. Zanfrognini, R. Cagnoli, A. Mucci, F. Parenti, L. Schenetti and G. Ponterini, Thin Solid Films, 2008, 516, 8731-8735.

34 B. Valeur and M. N. Berberan-Santos, Molecular Fluorescence. Principles and Applications, Wiley-VCH, Weinheim, 2013.

35 O. Horvát, R. Huszánk, Z. Valicsek and G. Lendvay, Coord. Chem. Rev., 2006, 250, 1792-1803.

36 Z. Valicsek and O. Horvát, Microchem. J., 2013, 107, 47-62.

37 I. Langmuir, J. Am. Chem. Soc., 1918, 40, 1361-1402.

38 M. Suzuki, Adsorption Engineering, Kodansha, Tokyo, 1990.

39 M. Haerifar and S. Azizian, J. Phys. Chem. C, 2013, 117, 83108317.

40 Y. S. Ho and G. McKay, Process Biochem., 1999, 34, 451-465.

41 J. Febrianto, A. N. Kosasih, J. Sunarso, Y.-H. Yu, N. Indranswati and S. Ismadji, J. Hazard. Mater., 2009, 162, 616-645.

42 Y. Liu and L. Shen, Langmuir, 2008, 24, 11625-11630.

43 W. Rudzinski and W. Plazinski, J. Phys. Chem. B, 2006, 110, 16514-16525.

44 W. Rudzinski and W. Plazinski, Adsorption, 2009, 15, 181192.

45 J. R. Lakowicz, Principles of Fluorescence Spectroscopy, Springer, New York, 2006.

46 R. Sips, J. Chem. Phys., 1948, 16, 490-495. 